diabetic control, these findings suggest that rapid strict diabetic control may trigger the processes causing retinopathy.

We thank Dr D P Mikhailidis and Mr M A Barradas for platelet studies; $M r J$ Y Jeremy and Dr R Hutton for thromboxane $B_{2}, \beta$ thromboglobulin, and platelet factor 4 measurements; and Mrs Pamela Dale for preparing the manuscript.

1 Van Ballegooie E, Hooymans JMH, Timmerman Z, et al. Rapid deterioration of retinopathy during treatment with continuous subcutaneous infusion. Diabetes

2 Lorenzi M, Goldbaum MH, Spencer EM, Cheney C. Improved diabetic control and retinopathy. New Engl $\mathcal{F}$ Med 1983;308:1600.

Kohner EM, Hamilton AM, Saunders S, Sutcliffe BA, Bulpitt J. The retinal blood flow in diabetes. Diabetologia 1970;11:27-33

4 Mikhailidis DP, Barradas MA, Jeremy JY, Mohiuddin J, Gracey L, Dandona P. Endogenous platelet thromboxane $A_{2}$ production in diabetic patients with and without peripheral vascular disease. Diabetologia 1983;25:180-1.

Rosove MH, Frank HJL, Harwig SSL. Plasma $\beta$-thromboglobulin, platelet factor 4, librinopeptide A, and other haemostatic function during improped
glycemic control in diabetes mellitus. Diabetes Care 1984;7:174-9.

(Accepred 5 December 1984)

Metabolic Unit, Department of Chemical Pathology and Human Metabolism, and Department of Ophthalmology, Royal Free Hospital and School of Medicine, London NW3 2QG

P DANDONA, DPHIL, MRCP, senior lecturer and director of metabolic unit J P BOLGER, MB, FRCS, senior registrar

F BOAG, MB, MRCP, registrar

V FONESCA, MD, MRCP, research fellow

J D ABRAMS, DM, FRCS, consultant ophthalmic surgeon

Correspondence to: Dr P Dandona.

\section{Peripheral neuropathy during treatment with almitrine}

Almitrine bismesylate $60 \mathrm{mg}$ daily is used widely in cerebrovascular disorders and is reportedly free of neurological side effects. ${ }^{1}$ At higher doses (100-150 mg daily) almitrine improves gas exchange in chronic obstructive pulmonary disease. ${ }^{2}$ To date no peripheral neuropathy has been related to treatment with almitrine. Since June 1984, however, we have seen four patients with chronic obstructive pulmonary disease who developed distal symmetrical sensory polyneuropathy during treatment with this drug.

\section{Case histories}

The table gives the relevant details of the patients. All patients had been receiving almitrine (100-150 mg daily) for four to seven months when they started to complain of paraesthesia and pain in distal lower extremities. A mild symmetrical distal sensory polyneuropathy was found on examination. Results of electrophysiological investigations were consistent with distal axonal loss. Morphological study of nerve specimens taken by biopsy of the superficial branch of the peroneal nerve showed axonal degeneration of an average of $20 \%$ (range $12-31 \%$ ) of the fibres isolated by teasing. Examination of cross sections showed moderate reduction of the density of myelinated fibres. On electron microscopy the unmyelinated fibres looked normal. Several clusters of regenerating myelinated fibres were observed. Morphological evidence suggested distal axonopathy in all four cases.

In no case could any other cause of the neuropathy be found-for example, metabolic disorders (diabetes mellitus or renal insufficiency), vitamin deficiency, neoplastic disease, gammopathy, exposure to toxic agents, or administration of any drug known occasionally to induce peripheral neuropathy. ${ }^{3}$

Almitrine was discontinued and the patients re-examined three months later. In every case the patient's condition had improved: sensory symptoms were less severe and the degree of sensory impairment was less.

\section{Comment}

Peripheral neuropathy with low nerve conduction velocity has been observed in chronic obstructive pulmonary disease and malnutrition ${ }^{4}$ but the finding has not been substantiated, and chronic obstructive pulmonary disease is not considered as a cause of neuropathy in recent textbooks. ${ }^{5}$ Each of our patients developed a sensory polyneuropathy within four to seven months after beginning treatment with almitrine bismesylate, and their condition started to improve within one to two months after withdrawal of the drug; this was in keeping with the axonal regeneration found in nerve biopsy samples. Neuropathy appeared to develop only in a minority of patients treated with almitrine, possibly as a consequence of some latent metabolic defect.

The French National Committee on Safety of Medicines and the manufacturers of the drug (Euthérapy, Neuilly) have been informed of our findings.

We thank Dr J L Renard, and Dr L Saporta for referring the patients.

1 Berthaux $P$, Laforestrie $R$. Mise en évidence de l'activité thérapeutique du 5023 SE sur l'efficacité intellectuelle et psycho-motrice des sujets âgés 1983;12:852-5.

2 Stradling JR, Nicholl CG, Cover D, et al. Pattern of breathing and gas exchange following oral almitrine bismesylate in patients with chronic obstructive pul-

3 Argov Z, Mastaglia FL. Drug-induced peripheral neuropathies. $\mathrm{Br}$ Med $\mathcal{f} 1979$;

4 Appenzelier D, Parks RD, MacGee J. Peripheral neuropathy in chronic disease

, Thomas PK, Lambert EH, Bunge R. Peripheral neuropathy. 2nd ed. . WB Saunders Company, 1984.

(Accepted 19 February 1985)

Centre Hospitalier de Meaux, 77104 Meaux, France

F CHEDRU, MD, head of neurology unit

R NODZENSKI, MD, fellow

J F DUNAND, MD, assistant

Hopital R Ballanger, 93602 Aulnay Sous Bois

G AMARENCO, MD, consultant physician

R GHNASSIA, MD, assistant

Centre Hospitalier de Bicetre, 94270 Le Kremlin-Bicetre

C CIAUDO-LACROIX, MD, assistant professor

G SAID, MD, associate professor

Correspondence to: Dr F Chedru.

Details of cases

\begin{tabular}{|c|c|c|c|c|c|c|c|c|c|c|}
\hline \multirow[b]{2}{*}{$\begin{array}{l}\text { Case } \\
\text { No }\end{array}$} & \multirow[b]{2}{*}{$\begin{array}{l}\text { Age } \\
\text { and } \\
\text { sex }\end{array}$} & \multirow[b]{2}{*}{$\begin{array}{c}\text { Daily dose of } \\
\text { almitrine } \\
\text { bismesylate } \\
\text { (mg) }\end{array}$} & \multicolumn{2}{|c|}{ Duration of treatment (months) } & \multirow[b]{2}{*}{ Other drugs } & \multirow[b]{2}{*}{$\begin{array}{c}\text { Main } \\
\text { clinical features }\end{array}$} & \multirow[b]{2}{*}{ Sensory complaints } & \multirow[b]{2}{*}{ Neurological signs* } & \multirow[b]{2}{*}{ Cerebrospinal fluid } & \multirow[b]{2}{*}{$\begin{array}{c}\text { Nerve } \\
\text { conduction } \\
\text { velocity } \dagger\end{array}$} \\
\hline & & & $\begin{array}{c}\text { Before first } \\
\text { sensory } \\
\text { complaint }\end{array}$ & $\begin{array}{c}\text { At time } \\
\text { of } \\
\text { examination }\end{array}$ & & & & & & \\
\hline 1 & $61 \mathrm{M}$ & 100 & 7 & 13 & $\begin{array}{c}\text { Theophylline, } \\
\text { oxygen }\end{array}$ & $\begin{array}{l}\text { Chronic obstructive } \\
\text { pulmonary disease }\end{array}$ & $\begin{array}{l}\text { Hyperaesthesia, } \\
\text { dysaesthesia, pain } \\
\text { (feet and legs) }\end{array}$ & $\begin{array}{l}\mathrm{T}, \mathrm{t}, \mathrm{P}= \pm(\mathrm{knees}) ; \\
\mathrm{PS}=0 \text { (toes); } \mathrm{V}=0 \\
\text { (iliac crests); no } \\
\text { motor deficit; ankle } \\
\text { reflexes absent }\end{array}$ & $\begin{array}{l}\text { Protein } 0.43 \mathrm{~g} / 1 \\
\text { cells } 5 \times 10^{6} / 1\end{array}$ & $\begin{array}{l}\text { Motor } 42 \mathrm{~m} / \mathrm{s} ; \\
\text { sensory not } \\
\text { measurable }\end{array}$ \\
\hline 2 & $61 \mathrm{M}$ & 150 & 5 & 12 & $\begin{array}{c}\text { Theophylline, } \\
\text { lorazepam }\end{array}$ & $\begin{array}{l}\text { Chronic obstructive } \\
\text { pulmonary disease, } \\
\text { neurotic depression, } \\
\text { recent weight loss } \\
(-5 \mathrm{~kg})\end{array}$ & $\begin{array}{l}\text { Paraesthesiae (upper } \\
\text { and lower limbs), } \\
\text { pain (legs and feet) }\end{array}$ & $\begin{array}{l}\mathrm{T}, \mathrm{t}, \mathrm{P}= \pm(\mathbf{k n e e s}) ; \\
\mathrm{PS}= \pm(\text { toes); } \mathrm{V}=0 \\
\text { (knees); no motor } \\
\text { deficit; ankle reflexes } \\
\text { absent }\end{array}$ & $\begin{array}{l}\text { Proteins } 0.27 \mathrm{~g} / 1 ; \\
\quad \text { cells }<1 \times 10^{\circ} / 1\end{array}$ & $\begin{array}{l}\text { Motor } 45 \mathrm{~m} / \mathrm{s} ; \\
\text { sensory } 32 \mathrm{~m} / \mathrm{s}\end{array}$ \\
\hline 3 & $54 \mathrm{M}$ & 100 & 7 & 12 & $\begin{array}{l}\text { Salbutamol, } \\
\text { cimetidine, } \\
\text { diltiazem, } \\
\text { isosorbide } \\
\text { dinitrate }\end{array}$ & $\begin{array}{l}\text { Chronic obstructive } \\
\text { pulmonary disease, } \\
\text { recent myocardial } \\
\text { infarction, peptic } \\
\text { ulcer, prostatic } \\
\text { adenoma }\end{array}$ & $\begin{array}{l}\text { Cramps, dysaesthesia } \\
\text { (legs, soles of feet) }\end{array}$ & $\begin{array}{l}\mathrm{T}, \mathrm{t}, \mathrm{P}= \pm(\mathrm{knees}) ; \\
\mathrm{PS}=0 \text { (ankles); } \mathrm{V}=0 \\
\text { (iliac crests); mild } \\
\text { motor deficit (legs); } \\
\text { ankle reflexes absent }\end{array}$ & $\begin{array}{l}\text { Proteins } 0.63 \mathrm{~g} / 1 ; \\
\text { cells }<1 \times 10^{6} / 1\end{array}$ & $\begin{array}{c}\text { Motor } 42 \mathrm{~m} / \mathrm{s} \\
\text { sensory not } \\
\text { measurable }\end{array}$ \\
\hline 4 & $55 \mathrm{M}$ & 100 & 4 & 5 & Theophylline & $\begin{array}{l}\text { Chronic obstructive } \\
\text { pulmonary disease, } \\
\text { history of lung } \\
\text { tuberculosis } 10 \\
\text { years before }\end{array}$ & $\begin{array}{c}\text { Paraesthesia, pain } \\
\text { (feet) }\end{array}$ & $\begin{array}{l}\mathrm{T}, \mathrm{t}, \mathrm{P}= \pm(\text { feet }) \\
\mathrm{PS}=+; \mathrm{V}=0 \\
\text { (ankles); no motor } \\
\text { deficit; deep tendon } \\
\text { reflex }=+\end{array}$ & $\begin{array}{l}\text { Protein } 0.44 \mathrm{~g} / 1 \text {; } \\
\text { cells }<1 \times 10^{6} / 1\end{array}$ & $\begin{array}{l}\text { Motor } 44 \mathrm{~m} / \mathrm{s} ; \\
\text { sensory } 38 \mathrm{~m} / \mathrm{s}\end{array}$ \\
\hline
\end{tabular}

- Sensation of touch (T), temperature ( $t$ ), pain (P), postural sense (PS), and vibration (V) graded as: 0 (totally abolished); \pm (impaired); $+($ normal). 